\title{
Resolvin D2 protects against cerebral ischemia/reperfusion injury in rats
}

\author{
Gang Zuo ${ }^{\dagger}$, Dongping Zhang ${ }^{\dagger}$, Rutao Mu${ }^{\dagger}$, Haitao Shen, Xiang Li, Zhong Wang ${ }^{*}$, Haiying Li ${ }^{*}$ (D) and Gang Chen
}

\begin{abstract}
Cerebral ischemia/reperfusion (I/R) injury is a critical factor leading to a poor prognosis for ischemic stroke patients. $\omega-3$ fatty acid supplements taken as part of a daily diet have been shown to improve the prognosis of patients with ischemic stroke. In this study, we aimed to investigate the potential effects of resolvin D2 (RvD2), a derivative of $\omega-3$ fatty acids, and its possible advantage on cerebral I/R injury in rats. Cerebral I/R caused by middle cerebral artery occlusion and reperfusion (MCAO/R) was established in Sprague-Dawley rats. First, in rats fed a regular diet, the MCAO/R stimulus led to a significant decrease in endogenous production of RvD2. Exogenous supply of RvD2 via intraperitoneal injection reversed MCAO/R-induced brain injury, including infarction, inflammatory response, brain edema, and neurological dysfunction. Meanwhile, RvD2 reversed the MCAO/R-induced decrease in the protein level of GPR18, which has been identified as a receptor for RvD2, especially in neurons and brain microvascular endothelial cells (BMVECs). Furthermore, RVD2 exerted rescue effects on MCAO/R-induced neuron and BMVEC death. Moreover, GPR18 antagonist O-1918 could block the rescue effects of RvD2, possibly at least partially though the GPR18-ERK1/2NOS signaling pathway. Finally, compared with $\omega$-3 fatty acid supplements, RvD2 treatment had a better rescue effect on cerebral infarction, which may be due to the MCAO/R-induced decrease in 5-lipoxygense phosphorylation and subsequent RvD2 generation. In conclusion, compared with $\omega-3$ fatty acids, RvD2 may be an optimal alternative and complementary treatment for ischemic stroke patients with recanalization treatment.
\end{abstract}

Keywords: Ischemic stroke, Cerebral ischemia/reperfusion injury, Resolvin D2, w-3 fatty acid, GPR18, Neuron, Brain microvascular endothelial cell

\section{Introduction}

Ischemic stroke is a common cause of long-term disability and is the second leading cause of death worldwide. Recanalization therapy provokes cerebral ischemia/reperfusion (I/R) injury, including vascular permeability increase, blood-brain barrier (BBB) disruption, and brain edema [1, 2]. Brain microvascular endothelial cells (BMVECs), the main cells composing the BBB, are connected by tight junction proteins such as zonula occludens 1 (ZO-1) [3]. BMVECs represent main targets for the reactive oxygen species (ROS) and inflammatory response produced during cerebral I/R [4]. Neuronal apoptosis and necrosis are other central pathological processes in I/R-induced brain injury. Inflammatory cytokines such as tumor necrosis factor $\alpha$ (TNF- $\alpha)$ and

\footnotetext{
*Correspondence: 15716201037@163.com; Ihy1015@suda.edu.cn

${ }^{\dagger}$ Equal contributors

Department of Neurosurgery \& Brain and Nerve Research Laboratory, The

First Affiliated Hospital of Soochow University, 188 Shizi Street, Suzhou 215006, China
}

interleukin 6 (IL-6), which are stimulated after necrosis induction, significantly increase in ischemic and reperfusion-induced brain tissue inflammation [5]. Therefore, the protection of BMVECs and neurons against I/R-induced oxidative stress and inflammatory response may be a potential therapeutic target for ischemic stroke patients with recanalization treatment [4].

Resolvin D2 (RvD2), a member of the resolvin family, is produced from $\omega-3$ polyunsaturated fatty acids after a series of catalyzed reactions by lipoxygenases [6]. Notably, $\omega-3$ fatty acids are able to cross the BBB effectively via its receptors to enter brain tissue [7]. It has been shown that both $\mathrm{RvD} 2$ and resolvin $\mathrm{D} 1$ can regulate the chronic inflammatory process that occurs in the adipose tissue of obese patients [8]. Another study found a direct correlation between the cerebrospinal fluid (CSF) levels of $\mathrm{RvD} 2$ in the post-mortem brain and the cognitive scores of patients with Alzheimer's disease [9]. In addition, aging rats treated with DHA were found to exhibit improved 
memory, which was accompanied by increased levels of RvD2 in the brain [9, 10]. Moreover, the antiinflammatory action of RvD2 is dependent on its G protein-coupled receptor (GPCR), GPR18, the mechanisms of which have not yet been elucidated [11, 12].

Advanced research has reported that fish oil taken as a supplement apart from daily diet may improve the clinical outcomes of patients with ischemic stroke. One study indicated that oral $\omega-3$ fatty acids could reduce cerebral infarction and the frequency of symptomatic vasospasm, and could also improve clinical prognosis [13]. In addition, our previous study found that $\omega-3$ fatty acids could protect early brain injury (EBI) from subarachnoid hemorrhage (SAH) [14]. The human body converts $\omega-3$ fatty acids into RvD2 through the lipoxygenase (LOX) metabolism: 12/15-lox can promote DHA conversion to 17-HDHA, and 17-HDHA then generates 17-HPDHA, which can be metabolized to RvD2 in a 5-LOX phosphorylation-dependent manner [15].

However, no study has yet reported the effects of RvD2 on I/R-induced brain injury. Therefore, the aim of this study was to investigate the effects of RvD2 on I/Rinduced brain injury in a rat $\mathrm{MCAO} / \mathrm{R}$ model as well as its underlying mechanisms.

\section{Results}

MCAO/R led to a significant decrease in the endogenous production of RvD2

In order to elucidate the endogenous production of RvD2 after I/R, we detected the RvD2 level in brain tissue after $\mathrm{MCAO} / \mathrm{R}$ by enzyme-linked immunosorbent assay (ELISA) in rats fed a regular diet. The results revealed that the endogenous RvD2 levels were significantly decreased in the $\mathrm{MCAO} / \mathrm{R}$ group compared with the sham group (Fig. 1a). At MCAO (2 h)/R (48 h), the levels of RvD2 had a restorative effect.

\section{Exogenous supply of RvD2 reversed MCAO/R-induced brain injury}

Next, we used exogenous RvD2 $(25,50$, and $100 \mu \mathrm{g} / \mathrm{kg})$ to treat the MCAO/R rats and detected its effects on inflammatory cytokine production and brain injury after $\mathrm{MCAO} / \mathrm{R}$. We found that the levels of IL- 6 and TNF- $\alpha$ in infarct-side brain tissue were significantly increased after $\mathrm{MCAO} / \mathrm{R}(72 \mathrm{~h})$, but were significantly decreased by $50 \mu \mathrm{g} / \mathrm{kg}$ and $100 \mu \mathrm{g} / \mathrm{kg} \mathrm{RvD2}$ treatment (Fig. 1b and c). Triphenyltetrazolium chloride (TTC) staining showed that the infarct area significantly decreased with 50 and $100 \mu \mathrm{g} / \mathrm{kg}$ RvD2 treatment (Fig. 1d and e). Compared with the sham group, the neurological score of the MCAO/R group was significantly higher, suggesting a remarkable neurological defect induction by the I/R model. However, the neurological scores of the MCAO/R + RvD2 (50 and $100 \mu \mathrm{g} / \mathrm{kg}$ ) groups were significantly lower than that of the $\mathrm{MCAO} / \mathrm{R}$ group (Fig. 1f). Additionally, brain water content was found to be significantly lower in infarct-side brain tissue samples of the RvD2 treatment group than in vehicle group rats, suggesting that RvD2 treatment effectively rescues the $\mathrm{MCAO} / \mathrm{R}$-induced $\mathrm{BBB}$ leakage (Fig. 1g). These findings were further confirmed by Western blot assays of brain albumin content (Fig. 1h). These results were consistent with the effects of RvD2 on inflammatory cytokine production.

\section{RvD2 reversed the MCAO/R-induced decrease in the} protein level of GPR18, especially in neurons and BMVECs To identify the mechanism underlying the neuroprotective effect of RvD2, we first examined the protein level of GPR18 which has been identified as a receptor for RvD2. Western blot analysis showed that the protein level of GPR18 was down-regulated in the $\mathrm{MCAO} / \mathrm{R}$ group compared to the sham group, whereas $50 \mu \mathrm{g} / \mathrm{kg}$ RvD2 treatment could reverse the decrease of GPR18 (Fig. 2a and b). The changes in GPR18 were consistent with results of previous experiments [16]. To further distinguish the cell type specificity of GPR18 expression, a Western blot assay was performed on cultured neurons, microglia, astrocytes, and brain microvascular endothelial cells (BMVECs). The results showed that GPR18 was mainly expressed in neurons, microglia, and BMVECs under normal conditions, with very little expression in astrocytes under both normal and OGD/R conditions (Fig. 2c and d). Compared with the normal group, the protein levels of GPR18 in neurons and BMVECs showed about a 3.5 -fold decrease at $72 \mathrm{~h}$ after OGD/R, while OGD/R induced only a slight decrease in the protein level of GPR18 in microglia (Fig. 2c and d). In addition, the OGD/R-induced decrease of GPR18 in neurons and BMVECs was reversed by RvD2 treatment, while RvD2 treatment did not induce a significant change in the protein level of GPR18 in microglia exposed to OGD/R (Fig. 2c and d). Based on these results, we focused on the roles of GPR18 in neurons and BMVECs in the following study.

\section{RvD2 exerted rescue effects on MCAO/R-induced neuron and BMVEC death}

We further explored the effects of RvD2 (25, 50, and100 $\mu \mathrm{g} / \mathrm{kg}$ ) on cell death in the ischemic penumbra of brain tissues at $72 \mathrm{~h}$ after MCAO/R by TUNEL and FJB staining. Compared with the sham group, TUNEL-positive neurons were significantly increased in the $\mathrm{MCAO} / \mathrm{R}$ group, while the numbers of TUNEL-positive neurons were reduced with RvD2 treatments (Fig. 3a and b). In addition, at $72 \mathrm{~h}$ after MCAO/R + RvD2 $(50 \mu \mathrm{g} / \mathrm{kg}$ and $100 \mu \mathrm{g} / \mathrm{kg}$ ), TUNEL-positive neurons were most obviously decreased compared with the $\mathrm{MCAO} / \mathrm{R}+\mathrm{RvD} 2$ $(25 \mu \mathrm{g} / \mathrm{kg})$ group. Consistently, the apoptotic index of 


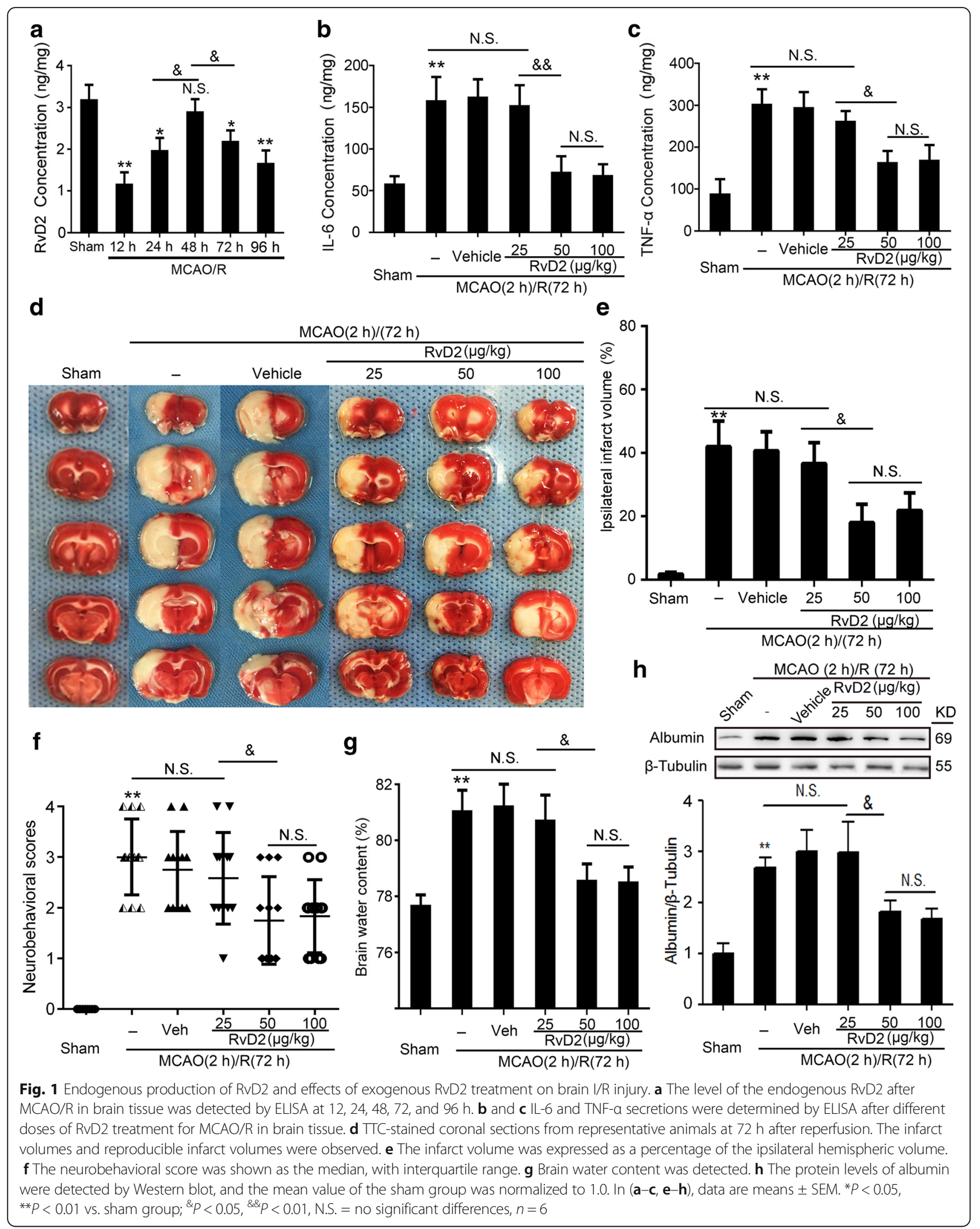




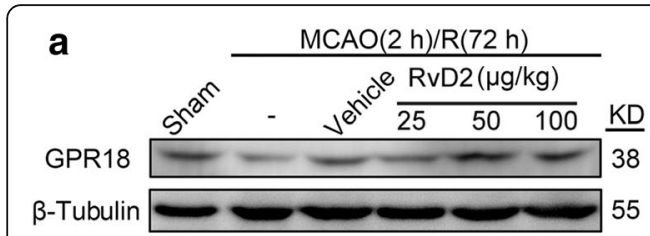

b

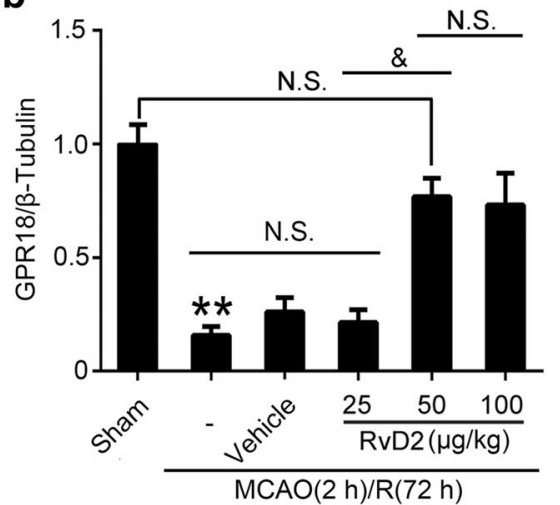

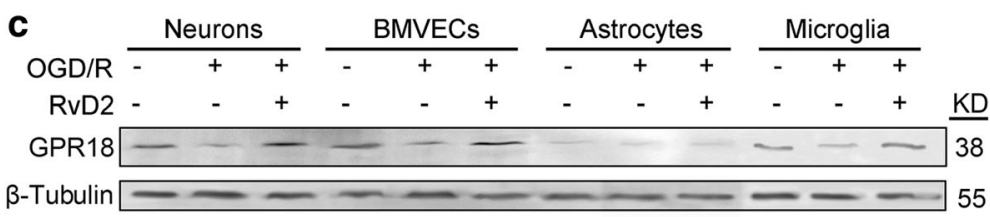

d

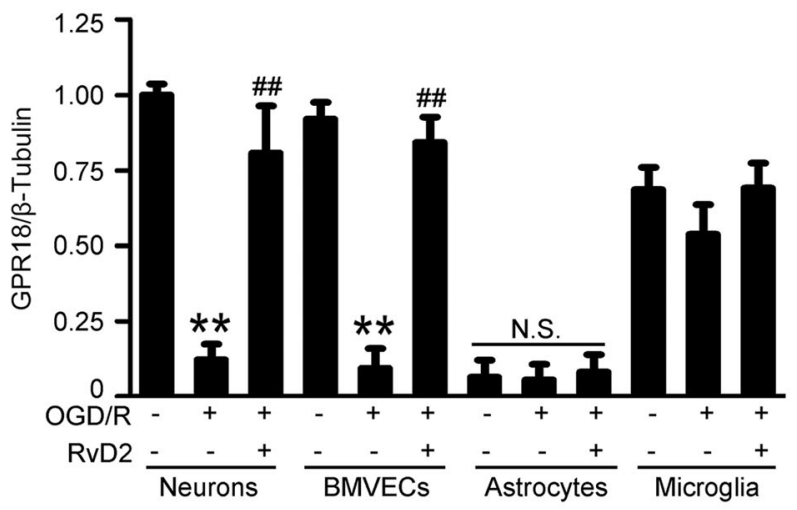

Fig. 2 RvD2 reversed the MCAO/R-induced decrease in the protein level of GPR18. a Western blot analysis was used to measure the protein levels of GPR18 after I/R brain injury. b The GPR18 expression levels were quantified. Data are presented as mean \pm SEM, $n=6 .{ }^{* *} P<0.01$ vs. sham group, ${ }^{*} P<0.05$. N.S. = no significant differences. c Western blot analysis was used to measure the protein levels of GPR18 in cultured neurons, astrocytes, microglia, and BMVECs under indicated treatments. $\mathbf{d}$ The GPR18 expression levels were quantified. The level of GPR18 in the normal neuron group was normalized to 1.0. Data are presented as mean $\pm \mathrm{SEM}, n=3$. ${ }^{* *} P<0.01 \mathrm{vs}$. normal group ${ }^{\# \#} P<0.01$. N.S. $=$ no significant differences

BMVECs showed the same trend (Fig. 3c and d). Meanwhile, FJB-positive cells in the $\mathrm{MCAO} / \mathrm{R}$ group were significantly increased compared with the sham group, while the number of FJB-positive brain cells was dramatically reduced in the RvD2 (50 and $100 \mu \mathrm{g} / \mathrm{kg}$ ) group at $72 \mathrm{~h}$ after I/R (Fig. 3e and f).

The neuroprotective effects of RvD2 were dependent on its receptor GPR18

Furthermore, we used O-1918, an antagonist of GPR18, to detect the mechanism underlying the neuroprotective function of RvD2. TTC staining showed that infarct volume was significantly increased in the RvD2 + O-1918 group compared to the RvD2 group under I/R conditions (Fig. 4a and b), which indicated that the neuroprotective effects of RvD2 were dependent on GPR18.

RvD2 exhibited rescue effects through the GPR18-ERK1/2NOS signaling pathway

Next, we found that RvD2 promoted ERK1/2 phosphorylation, which was inhibited by O-1918 treatment (Fig. 5a and b). In addition, the protein levels of neuronal NOS (nNOS) and endothelial NOS (eNOS) were significantly abated in the ischemic hemisphere after MCAO/R (72 h). Meanwhile, the production of nNOS, eNOS, and ZO-1 were significantly enhanced after treatment with RvD2 at $72 \mathrm{~h}$ after reperfusion (Fig. $5 \mathrm{c}-\mathrm{l}$ ). And, the O-1918 treatment could partly block the function of RvD2 (Fig. 5).
RvD2 exerted a better rescue effect on cerebral infarction than $\omega-3$ fatty acids

Because $\omega-3$ fatty acids are precursors of RvD2, we also compared the different effects of injecting RvD2 directly and supplying $\omega 3$-polyunsaturated fatty acids. TTC staining was performed to observe the infarct area of the ischemic hemisphere, and we found that the volume of the ischemia was significantly decreased after the RvD2 or $\omega-3$ treatment, while RvD2 seemed to be more effective at protecting I/R against infarction (Fig. 6a and b).

\section{MCAO/R induced a decrease in 5-LOX phosphorylation} and subsequent RvD2 generation

In the sham group, supplementation of $\omega-3$ fatty acids promoted RvD2 production, whereas the RvD2 synthesis was restrained after $\mathrm{MCAO} / \mathrm{R}$, indicating that the effects of $\omega-3$ fatty acids were disabled after MCAO/R (Fig. 6c). Because LOX activity was involved in converting $\omega-3$ into RvD2, we determined the phosphorylation of 5-LOX in the brain by Western blot. Phosphorylated 5-LOX in brain tissue was much lower than that in the sham group after MCAO/R (Fig. 6d and e), which was in accordance with the change of RvD2 production after $M C A O / R$ as shown in Fig. 1a. These results indicated that $\mathrm{MCAO} / \mathrm{R}$-induced a decrease in 5-LOX phosphorylation and subsequent RvD2 generation.

\section{Discussion}

Cerebral ischemia is one of the main causes of death around the world. The clinical treatment of cerebral 


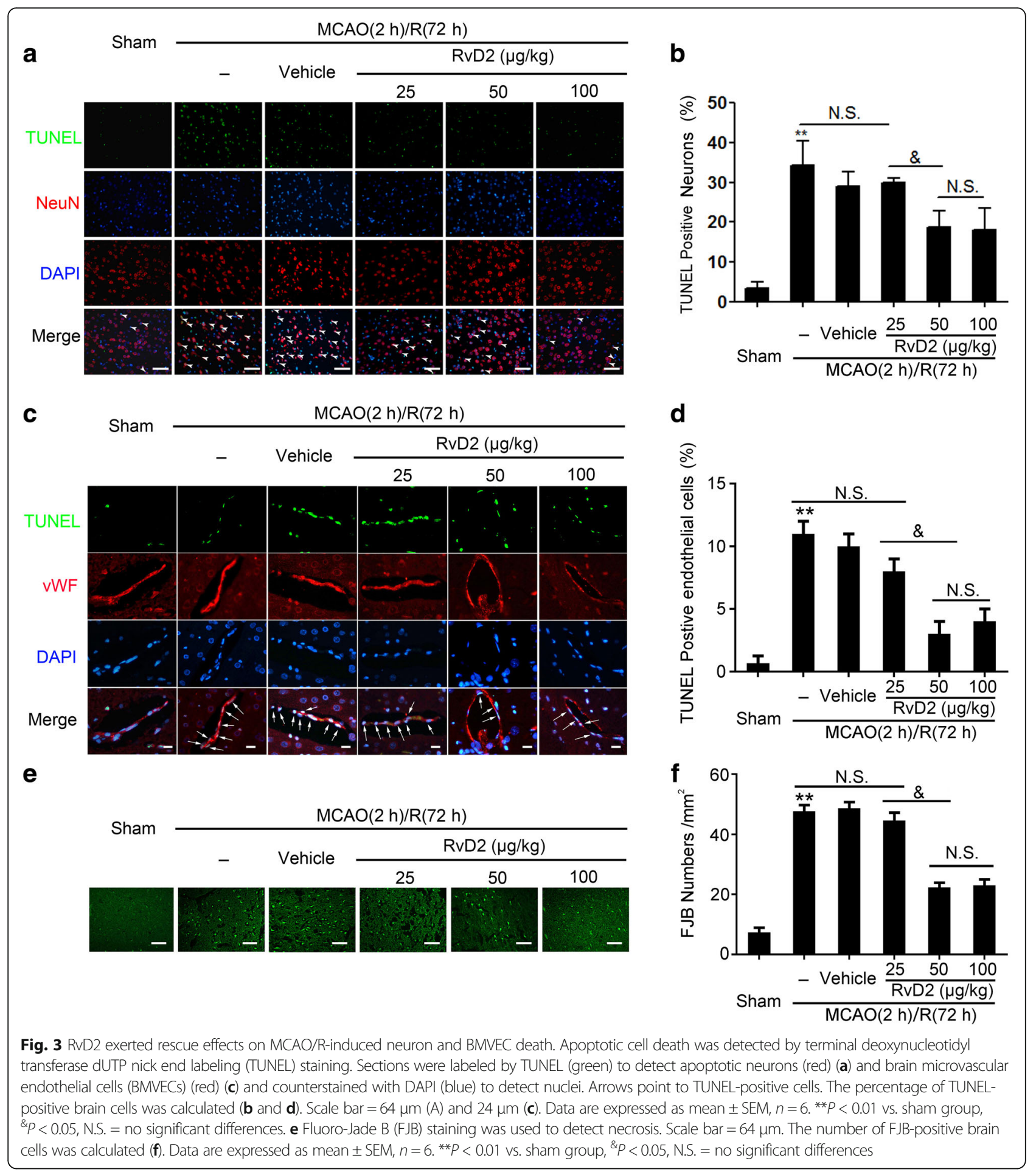

ischemia is limited, and new neuroprotective therapies are desperately needed. In the present study, we found that the generation of endogenous RvD2 was decreased and the supplementation of exogenous RvD2 played a role in neuroprotection after $\mathrm{MCAO} / \mathrm{R}$. To date, information on the generation and function of RvD2 in the brain was very limited.
The existence of RvD2 was discovered in the brains of mice subjected to stroke in a study aimed to develop methods for the detection of resolvins, indicating that it could be involved in the improvement of ischemic lesions [17]. It was reported that RvD2 could reverse LPS-induced depression-like behaviors through the mTORC1 signaling pathway [18]. Consistently, our 

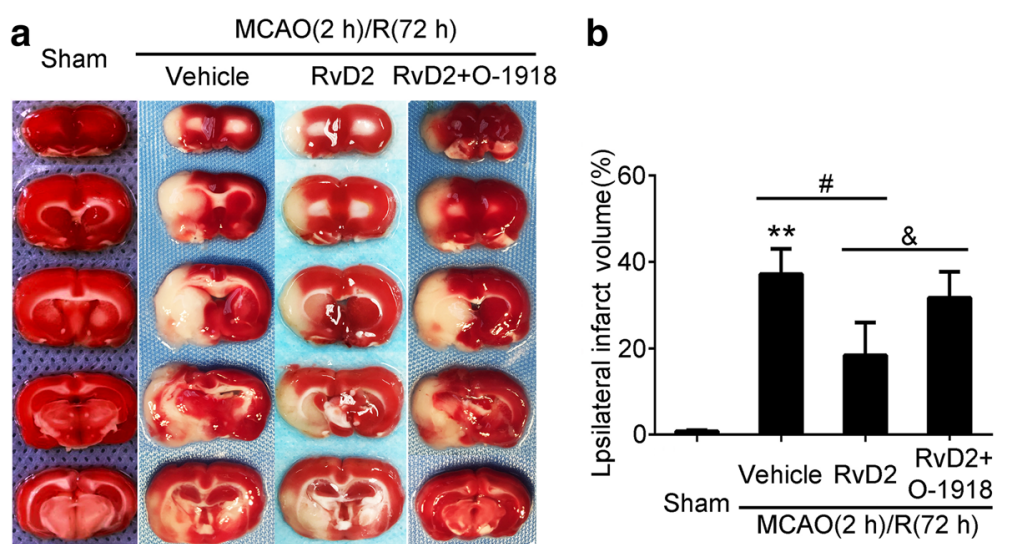

Fig. 4 The rescue effects of RvD2 were reversed by GPR18 antagonist O-1918 after MCAO/R. a TTC-stained coronal sections from representative animals at $72 \mathrm{~h}$ after reperfusion. $\mathbf{b}$ Statistical analysis of infarct volumes. The infarct volume was expressed as a percentage of the ipsilateral hemispheric volume. Data were means \pm SEM. ${ }^{*} P<0.05$, ${ }^{*} P<0.01$ vs. sham group, ${ }^{\#} P<0.05$, ${ }^{\circledR} P<0.05, n=6$

results confirmed that a cessation of the generation of resolvin in the brain could be related to the brain injury after ischemic stroke. Therefore, exogenous RvD2 was able to reduce the release of TNF- $\alpha$ and IL6 in the brain, decrease the infarct area, and protect the neurons and BMVECs cells from apoptosis and necrosis after $\mathrm{MCAO/R}$ (Fig. 7). Although previous studies reported the protective effects of RvD2 on brain regions [10, 19], to our knowledge, these are the first reported results to suggest that RvD2 acts on I/R brain injury.

Moreover, the possible mechanisms of the action of RvD2 have not been clarified. In 2015, Nan et al. presented evidence that GPR18 was expressed on human leukocytes as a specific cell surface GPCR for RvD2 [20]. GPRs, composed of seven transmembrane domains, are the largest family of receptors and regulate various physiological processes. One of these receptors, GPR18, consists of 331 amino acids and is conserved in mammals, which was originally described in the human spleen and testes [21]. Moreover, the expressions and distributions of GPR18 were evaluated in the hypothalamus neurons on diet induced obesity. Additionally, GPR18 was found to be extensively distributed in the central nervous system (CNS), including in microglia, and play a significant role in the regulation of microglia in the CNS [22]. Another study, in which RvD2 was injected into the spine to treat chronic pain, suggested that sensory neurons are responsive to this resolvin and may express GPR18 [23]. As expected, we found that the expression of GPR18 was inhibited after MCAO/R, which was reversed by RvD2 treatment. In addition, the neuroprotective effects of RvD2 were significantly abolished by O-1918, which was identified as an antagonist for GPR18 as a structurally related synthetic compound. Therefore, we confirmed that RvD2 exerts its effects through GPR18 to transfer the signaling in neurons and
BMVEC cells. In our study, based on the cell type specificity of GPR18 expression shown in Fig. 2c and d, we focused only on the effects of $\mathrm{RvD} 2$ on neuron apoptosis and $\mathrm{BBB}$ maintenance. The mechanisms underlying the role of RvD2 in inflammation by GPR18 expression in microglia require further research.

It is known that ROS induces a series of oxidative stress reactions and leads to brain cell apoptosis and necrosis during $\mathrm{I} / \mathrm{R}$ stroke onset. NO can be produced by three different isoforms of the enzyme NOS to play the role of an antioxidant. nNOS is expressed in specific neurons of the CNS and is involved in synaptic plasticity. Among the NOSs, eNOS, as the most important isoform, can keep blood vessels dilated and control blood pressure, which has numerous vasoprotective and anti-atherosclerotic functions [24]. In the present study, nNOS and eNOS, considered anti-inflammation biosynthesizers, were significantly enhanced after RvD2 post-treatment in the MCAO/R model. After activation of GPR18, the synthesis of NOS could be controlled by the ERK1/2 pathway [25]. ERK plays an essential role in many cellular and physiological processes, including cell proliferation, differentiation, survival, migration, invasion, transcription, metabolism, and apoptosis. The expression of a subset of tight junction (TJ) proteins such as ZO-1 also depends on ERK1/2 phosphorylation [26]. In our study, we found that the decreases in ERK1/2 phosphorylation and ZO-1 expression were significantly ameliorated by RvD2 treatment after MCAO/R, resulting in improved nNOS and eNOS levels, thereby maintaining BBB integrity.

In addition, RvD2 was metabolized by $\omega-3$ fatty acids, especially DHA, which was separated from fish oil. In recent years, fish oil has been favored by both consumers and academics, and its brain protection in ischemic stroke has garnered a great deal of interest. Pretreatment with 


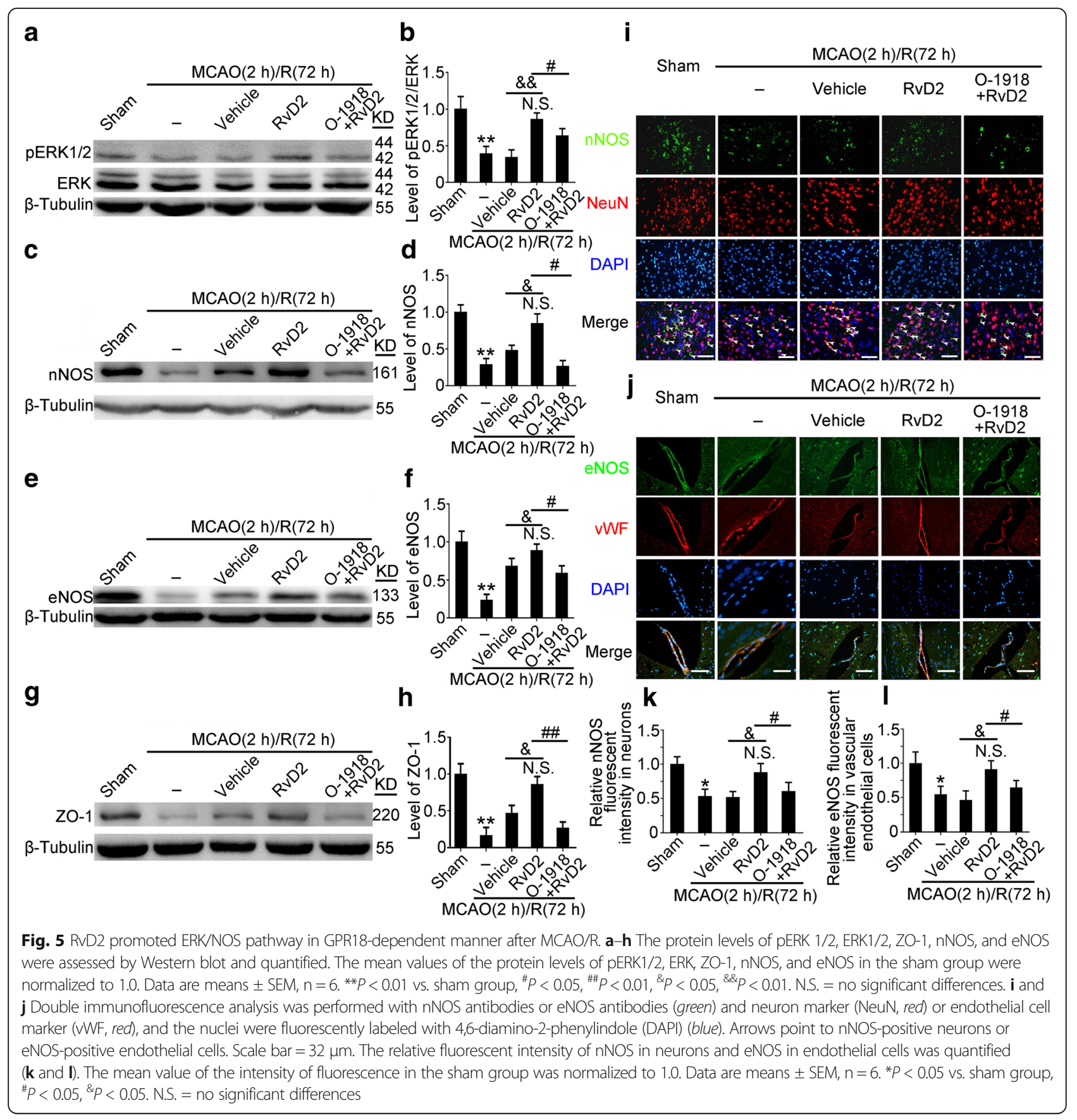

fish oil or its constituent $\omega-3$ fatty acids has been shown to alleviate I/R-induced brain injury [27-29], suggesting that fish oil can effectively reduce I/R damage. However, post-treatment is a much more likely scenario in clinical treatment. Correia et al. found that DHA pretreatment could effectively improve the memory recovery function of cerebral ischemia rats, but DHA postconditioning did not have the same effect [30]. Additionally, Yang found that DHA post-treatment increased rat brain I/R injury [31]. Here, we found that the capacity of $\omega-3$ fatty acids to generate $\mathrm{RvD} 2$ was reduced by $\mathrm{MCAO} / \mathrm{R}$, making them less effective than direct RvD2 injection, suggesting that in the early brain I/R injury process, the metabolic process of fish oil (especially DHA) may be blocked. Furthermore, LOX played a critical role in the conversion of DHA into RvD2: 12/15-lox could promote DHA conversion to 17-HDHA, and 17-HDHA then generated 17-HPDHA, which could be metabolized to RvD2 after 5-LOX phosphorylation. A recent study suggested that phosphorylation of 5-LOX at serine 663 could stimulate lipoxin production, which plays a role in the last steps of RvD2 synthesis [32]. In this study, we found that the level of 
a

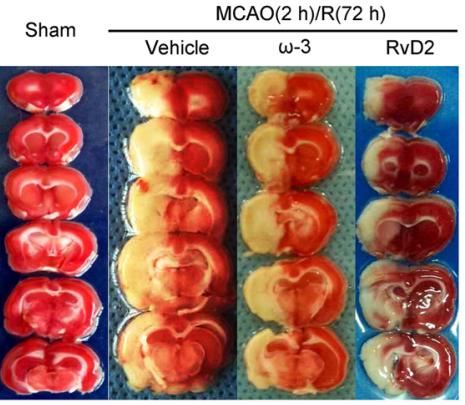

d

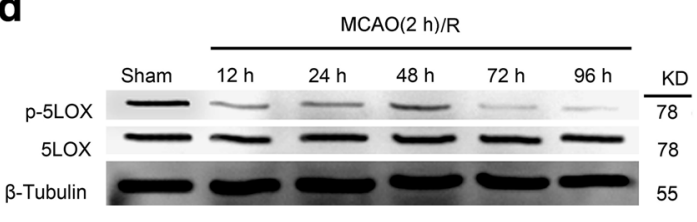

C

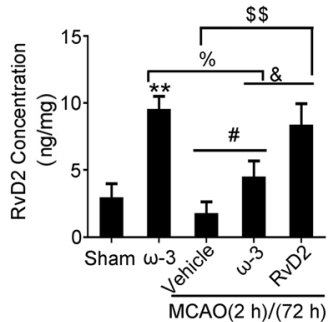

e

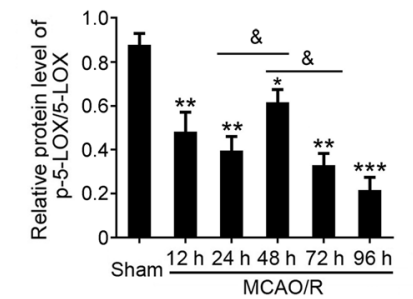

Fig. $6 \omega$-3 fatty acids was partly disabled after MCAO/R. a TTC-stained coronal sections from representative animals at $72 \mathrm{~h}$ after reperfusion. b Statistical analysis of infarct volume. Reproducible infarct volumes were observed in the MCAO/R + vehicle group, MCAO/R + $\omega-3$ group, and MCAO/R + RvD2 group. The infarct volume is expressed as a percentage of the ipsilateral hemispheric volume. Data are means \pm SEM. ${ }^{* *} P<0.01$ vs. sham group, ${ }^{\#} P<0.05$,

${ }^{\$} P<0.05,{ }^{\&} P<0.05, n=6 . \mathbf{c}$ RVD2 production after supplying $\omega-3$ fatty acids and injection of RvD2 was detected by ELISA. Data are means $\pm S E M, n=6$. ${ }^{*} P<0.01$ vs. sham group, ${ }^{\#} P<0.05,{ }^{\&} P<0.05,{ }^{\%} P<0.05$, ${ }^{\$} P P<0.01$. N.S. $=$ no significant differences. $\mathbf{d}$ Western blot analysis was used to detect the protein levels of 5-LOX and Phos-5-LOX after I/R brain injury. Quantification of the Phos-5-LOX/5-LOX level is shown (e). Data are presented as mean $\pm S E M, n=6$. ${ }^{* *} P<0.01,{ }^{* * *} P<0.001$ vs. sham group, ${ }^{\circledR} P<0.05$, N.S. $=$ no significant differences

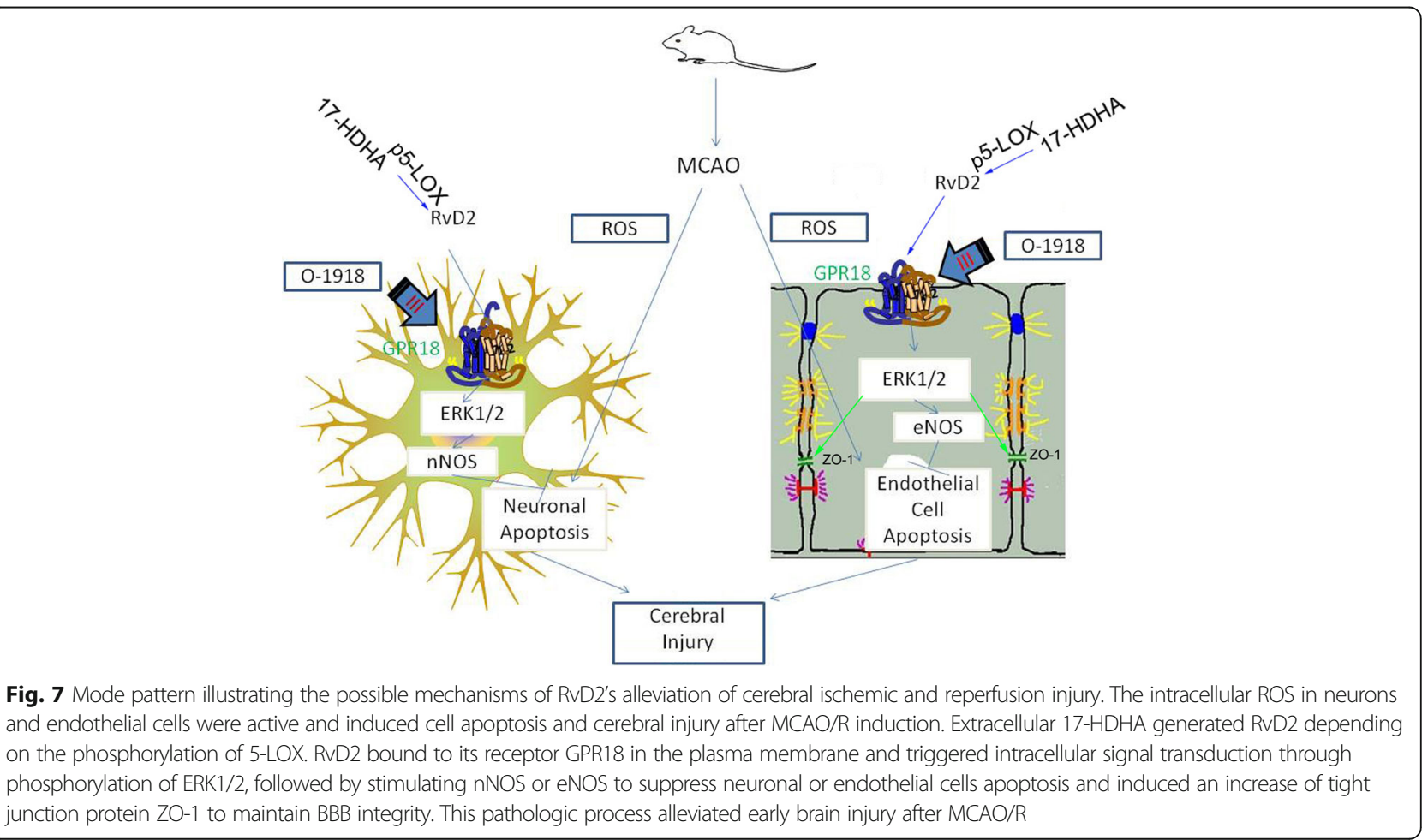


p5-LOX was significantly decreased after $\mathrm{MCAO} / \mathrm{R}$, leading to the metabolic failure of $\omega-3$ fatty acids.

This study demonstrated that the functions of $\omega-3$ fatty acids were partly blocked by the decrease in p5-LOX after I/R injury. For the first time, RvD2 was reported to alleviate cerebral I/R-induced brain injury, suggesting that it could be a novel neuroprotective candidate.

\section{Methods}

Animal and ethics approval and consent to participate Adult male Sprague-Dawley (SD) rats weighing 250-300 g were provided by the Animal Center of the Chinese Academy of Sciences, Shanghai, China. The rats were housed in animal quarters under controlled temperature and humidity with a $12 \mathrm{~h}$ light/dark cycle. All animal procedures are strictly in accordance with the guideline of the Ethics Committee of Soochow University on animal care and use.

\section{Rat model of cerebral $\mathrm{I} / \mathrm{R}$ and groups}

In experiment 1,36 adult male SD rats (42 rats were used, but only 36 rats survived after the MCAO modeling surgery) were randomly assigned into 6 groups, the sham group and the experimental groups arranged by time: $12,24,48,72$, and $96 \mathrm{~h}(n=6$ per group). Under an operating microscope, the focal cerebral ischemia was accomplished by right-sided endovascular middle cerebral artery occlusion (MCAO). Briefly, the rats were anesthetized, and the rectal temperature was maintained at $37 \pm 0.5{ }^{\circ} \mathrm{C}$ with a heating lamp. Then, the right common carotid arteries, external carotid arteries, and internal carotid arteries were revealed surgically. Next, a piece of 4-0-monofilament nylon suture with a blunted tip coated with polylysine was used to occlude the middle cerebral artery (MCA). The nylon suture was inserted through the right common carotid arteries and from the external carotid arteries into the internal carotid arteries until the tip occluded the proximal stem, indicating MCAO induction. After ischemia for $2 \mathrm{~h}$, the filament was withdrawn to allow reperfusion. At 12, 24, 48, 72, and $96 \mathrm{~h}$ of ischemia after reperfusion, brain tissues were obtained for analysis (Fig. 8a).

In experiment 2, 108 adult male SD rats (120 rats were used, 108 rats survived after the MCAO modeling
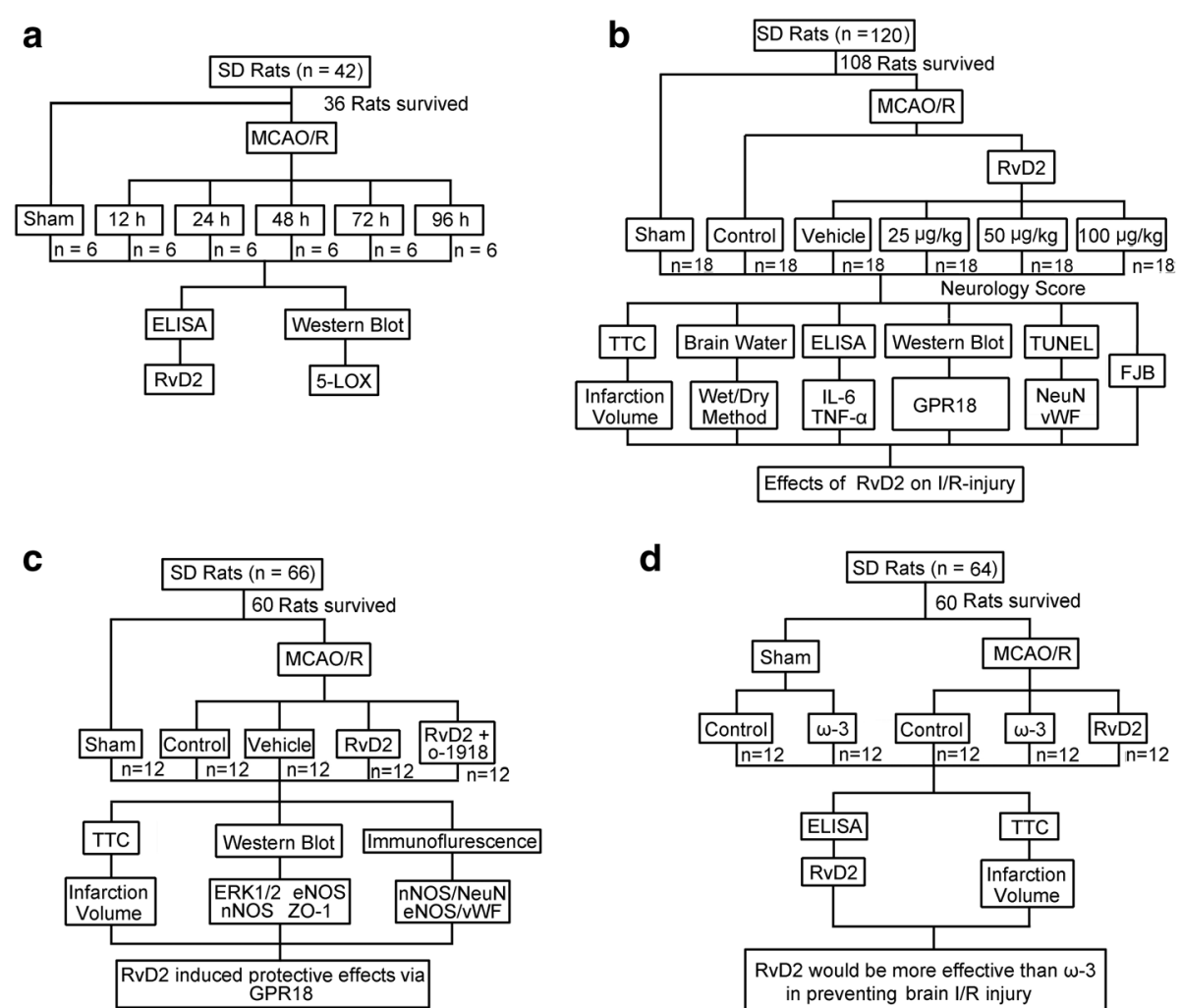

Fig. 8 Animal groups. a The first experiment was designed to show the time course of the protein level of 5-LOX, phosphorylation of 5-LOX, and levels of endogenous RvD2 after MCAO/R and identify the suitable time point for the second experiment. $\mathbf{b}$ The second experiment was designed to observe the effects of intervention with exogenous RvD2 at different doses on I/R brain injury after MCAO/R in SD rats. c The third experiment was designed to identify the potential mechanisms of the alleviation of I/R-brain injury by RvD2. $\mathbf{d}$ The fourth experiment was designed to compare the effects of RvD2 on I/R with those of $\omega 3$-polyunsaturated fatty acids in vivo 
surgery) were randomly divided into six groups: sham group, $\mathrm{MCAO} / \mathrm{R}$ group, $\mathrm{MCAO} / \mathrm{R}+$ vehicle group, $\mathrm{MCAO} / \mathrm{R}+\mathrm{RvD} 2(25 \mu \mathrm{g} / \mathrm{kg})$ group, $\mathrm{MCAO} / \mathrm{R}+\mathrm{RvD2}$ $(50 \mu \mathrm{g} / \mathrm{kg})$ group, and MCAO/R + RvD2 (100 $\mu \mathrm{g} / \mathrm{kg})$ group ( $n=18$ per group). After the indicated treatments, rats were euthanized, and the brain tissues were separated and taken for analysis (Fig. 8b).

In experiment 3, 60 adult male SD rats (66 rats were used, but only 60 rats survived after the MCAO modeling surgery) were randomly assigned into five groups: sham group, $\mathrm{MCAO} / \mathrm{R}$ group, $\mathrm{MCAO} / \mathrm{R}+$ vehicle group, $\mathrm{MCAO} / \mathrm{R}+\mathrm{RvD} 2(50 \mu \mathrm{g} / \mathrm{kg})$ group, and $\mathrm{MCAO} / \mathrm{R}+$ RvD2 $(50 \mu \mathrm{g} / \mathrm{kg})+\mathrm{O}-1918$ group ( $n=12$ per group). After the indicated treatments, rats were euthanized, and the brain tissues were separated and taken for analysis (Fig. 8c).

In experiment 4, 60 adult male SD rats (64 rats were used, 60 rats survived after the surgery) were divided into five groups: sham group, sham $+\omega-3$ group, $\mathrm{MCAO} / \mathrm{R}$ group, $\mathrm{MCAO} / \mathrm{R}+\omega-3$ group, and $\mathrm{MCAO} / \mathrm{R}$ $+\operatorname{RvD2}(50 \mu \mathrm{g} / \mathrm{kg})$ group $(\mathrm{n}=12$ per group). After the indicated treatments, rats were euthanized, and the brain tissues were separated and taken for analysis (Fig. 8d).

\section{Ischemic penumbra dissection}

After the indicated treatments, the rats were transcardially perfused with ice-cold PBS. Then the brain was quickly removed and frozen in stainless steel brain matrices $\left(-20{ }^{\circ} \mathrm{C}\right)$ for $10 \mathrm{~min}$, and the cerebellum and olfactory bulb were discarded. As described previously [33], the ischemic penumbra was dissected. In brief, the brain was sliced into three slices from the anterior tip of the frontal lobe. Both the front and back slices were sliced at $3 \mathrm{~mm}$ thickness, while the middle slice was sliced at $4 \mathrm{~mm}$ thickness. The middle slice was then cut in the ischemic hemisphere $2 \mathrm{~mm}$ from the midline longitudinally. To separate the core from the penumbra, a transverse diagonal cut was made at the 2 o'clock position. Finally, the cerebral cortical penumbra was harvested for Western blot and immunohistochemistry analyses.

\section{Extraction and culture of neurons}

Primary neurons were prepared from the brains of fetal rats (from 16 to 18-day pregnant SD rats). Every effort we made was to reduce the number of embryos used and their suffering. First, we removed the meninges and blood vessels. Next, the brains were digested with $0.25 \%$ trypsin (with ethylenediaminetetraacetic acid [EDTA]) for 5 min and washed with PBS 3 times to terminate the digestion. Then, we suspended the tissue in PBS, filtered the cell suspension, and centrifuged the suspension at $500 \times \mathrm{g}$ for $5 \mathrm{~min}$. The neurons were then suspended and plated on a poly-L-lysine-coated culture vessel and cultured in Neurobasal-A medium containing 2\% B27,
$2 \mathrm{mM}$ L-glutamine, $50 \mathrm{U} / \mathrm{ml}$ penicillin, and $50 \mathrm{U} / \mathrm{ml}$ streptomycin (all from Gibco, Carlsbad, CA, USA). Half of the medium was renewed every 2 days for 2 weeks. Finally, the neurons were exposed to the indicated treatments and harvested.

\section{Extraction and culture of microglia}

Primary microglia were prepared from brains of 1-dayold newborn rats. The digestion process was similar to that of neurons. After centrifugation, cell deposits were suspended in DMEM/F12 containing 10\% fetal bovine serum, $1 \mathrm{mM}$ sodium pyruvate, $2 \mathrm{mM} \mathrm{L}$-glutamine, $100 \mathrm{mM}$ nonessential amino acids, $50 \mathrm{U} / \mathrm{ml}$ penicillin, and $50 \mathrm{U} / \mathrm{ml}$ streptomycin (all from Gibco, Carlsbad, CA, USA). Then cells were seeded into a $150 \mathrm{~cm}^{2}$ culture flask in the fresh DMEM/F12 medium. Half of the medium was renewed every 2 days. Two weeks after initial seeding, a confluent polylayer of glial cells could be observed, and microglia could be separated from astrocytes by shaking the flask for $4 \mathrm{~h}$ at $150 \mathrm{rpm}$. The cell suspension was collected and centrifuged. Finally, the microglia were suspended and plated on poly-L-lysinecoated 12-well plates in the fresh medium.

\section{BMVEC culture}

The human cerebral microvascular endothelial cell line hCMEC/D3 was purchased from the Cell Bank of the Chinese Academy of Sciences (Shanghai, China). hCMEC/D3 cells were cultured as routine in 1640 medium (Hyclone, SH30809.01B) supplemented with $10 \%$ heat-inactivated fetal bovine serum.

\section{Astrocyte culture}

The human astrocyte cell line was obtained from ScienCell Research Laboratories (Cat \#1800). According to the manufacturer's instructions, astrocytes were plated on a poly-L-lysine-coated culture vessel and cultured in astrocyte medium (ScienCell, Cat \#1801).

\section{Antibodies}

Rabbit anti-GPR18 polyclonal antibody (SAB4501252) was provided by Sigma. Rabbit anti-albumin polyclonal antibody (ab51422), rabbit anti-eNOS polyclonal antibody (ab66127), rabbit anti-nNOS monoclonal antibody (ab76067), rabbit anti-5-LOX monoclonal antibody (ab169755), mouse anti-NeuN antibody (ab104224), mouse anti-GFAP antibody (ab10062), and mouse antivon Willebrand factor (vWF) antibody (ab68545) were purchased from Abcam (Cambridge, MA, USA). Rabbit anti-5-LOX (phospho-Ser663) polyclonal antibody (P12527) was purchased from Biorbyt (Cambridge, CA, UK). Rabbit anti-ERK1/2 (phospho-Thr202/Tyr204) antibody (\#4370) and monoclonal rabbit anti- $\beta$-tubulin antibody (\#2146) were provided by Cell Signaling 
Technology (Danvers, MA, USA). Rabbit anti-ZO-1 polyclonal antibody (RA231621) was purchased from Thermo Fisher (Waltham, MA, USA). Goat anti-rabbit IgG HRP (sc-2004) was purchased from Santa Cruz Biotechnology (CA, USA). Alexa Fluor-488 donkey antirabbit IgG antibody (A21206) and Alexa Fluor-555 donkey anti-mouse IgG antibody (A31570) were purchased from Invitrogen (Carlsbad, CA, USA).

\section{Reagents}

RvD2 (item no. 10007279) was purchased from Cayman (Ann Arbor, MI, USA). Fish oil (cholesterol free) containing 30\% $\omega-3$ fatty acids was obtained from Nature's Bounty, Inc.; $1000 \mathrm{mg}$ fish oil contained $300 \mathrm{mg} \omega-3$ fatty acids, including $180 \mathrm{mg}$ EPA and $120 \mathrm{mg}$ DHA. O-1918 (ab120405) was purchased from Abcam. All of the reagents for SDS-polyacrylamide gel electrophoresis and Western blot were purchased from Bio-Rad (Richmond, CA, USA). Phenylmethylsulfonyl fluoride (PMSF), aprotinin, Triton X-100, dithiothreitol (DTT), glycerol, Tween 20, BSA (fraction V), and TTC (T8877) were purchased from Sigma Chemical Co. (St. Louis, $\mathrm{MO}$, USA). Chemiluminescence protein labeling reagents in immunoblots were purchased from Thermo Fisher. The terminal deoxynucleotidyl transferase dUTP nick end labeling (TUNEL) staining kit (DeadEnd fluorometric kit) was purchased from Promega (Madison, WI, USA). Fluoro-Jade B (FJB) was purchased from Histo-Chem, Inc. (Jefferson, AR, USA), and 4',6-diamidino-2-phenylindole (DAPI) was obtained from SouthernBiotech (Birmingham, AL, USA).

\section{ELISA}

The brain concentrations of RvD2, IL- 6 , and TNF- $\alpha$ were determined by an RvD2 ELISA kit (Cayman, 501,120), rat IL-6 kit (USCN, Ra20607), and rat TNF- $\alpha$ kit (USCN, Ra20035). These assays were performed according to the manufacturers' instructions.

\section{Neurobehavioral scores}

At $72 \mathrm{~h}$ after MCAO/R, the behavioral impairment was evaluated utilizing a previously published scoring system, and the appetite, activity, and neurological defects of all rats were monitored in experiment 2 [34] (Table 1). All behavioral tests were performed by investigators who were blinded to the group assignment.

\section{Brain edema}

As described previously [35], the wet/dry method was used to evaluated the brain edema. Briefly, the brain tissues were collected, and the samples of brainstem and cerebellum were weighed immediately as the wet weight. The samples were kept at $100{ }^{\circ} \mathrm{C}$ for $72 \mathrm{~h}$ and then weighed again to obtain the dry weight. The percentage
Table 1 Behavior and activity scores

\begin{tabular}{lll}
\hline Category & Behavior & Score \\
\hline Appetite & Finished meal & 0 \\
& Left meal unfinished & 1 \\
& Scarcely ate & 2 \\
Activity & Walk and reach at least three corners of the cage & 0 \\
& Walk with some stimulations & 1 \\
& Almost always lying down & 2 \\
Deficits & No deficits & 0 \\
& Unstable walk & 1 \\
& Impossible to walk & 2 \\
\hline
\end{tabular}

of water content was calculated as follows: [(wet weight - dry weight)/wet weight] $\times 100 \%$.

\section{TUNEL and FJB staining}

First, all the paraffin sections $(4-6 \mu \mathrm{m})$ for detection were dried for $1 \mathrm{~h}$ at $70{ }^{\circ} \mathrm{C}$ and deparaffinized in xylene and graded ethanol solutions (100\%, 95\%, 90\%, 80\%, and 70\%).

According to the manufacturer's protocol, TUNEL staining was used to detect the cell apoptosis in brain tissue. Then, the TUNEL staining slides were incubated with von Willebrand factor (vWF) and NeuN antibody at $4{ }^{\circ} \mathrm{C}$ overnight, followed by the incubation of the indicated secondary antibody at $37{ }^{\circ} \mathrm{C}$ for $1 \mathrm{~h}$. Finally, the slides were visualized by a fluorescence microscope (Olympus BX50/BX-FLA/DP70), and the TUNELpositive cell count was obtained, which was defined as the average number of TUNEL-positive cells in each section counted in six microscopic fields.

FJB staining was used as a marker of neuronal injury and performed as follows. After deparaffinizing and rehydrating of the brain sections, the slides were incubated with $80 \%$ alcohol containing $1 \% \mathrm{NaOH}$ for $1 \mathrm{~min}$, followed by incubation in $0.06 \%$ potassium permanganate for $15 \mathrm{~min}$. Then, the slides were rinsed in deionized water and immersed in $0.001 \%$ FJB working solution $(0.1 \%$ acetic acid) for $30 \mathrm{~min}$ at room temperature, after which they were washed and dried in an incubator $\left(50-60{ }^{\circ} \mathrm{C}\right)$ for $10 \mathrm{~min}$. Then, the sections were dehydrated with xylene and covered by neutral balsam (Distyrene Plasticiser Xylene, Sigma-Aldrich, Inc., St. Louis, MO). The stained tissue sections were visualized by a fluorescence microscope, and the numbers of FJB positive cells per $\mathrm{mm}^{2}$ were counted in the selected fields.

\section{Western blot analysis}

Western blot analysis was conducted as described previously [36]. The brain samples were mechanically lysed in precooling RIPA lysis buffer (Beyotime) for $30 \mathrm{~min}$ and centrifuged at 12,000 rpm for $20 \mathrm{~min}$. After the supernatant was collected, the protein concentrations were 
determined by the bicinchoninic acid (BCA) protein assay kit (Beyotime). Protein samples $(50 \mu \mathrm{g})$ were separated by a $12 \%$ SDS-polyacrylamide gel and transferred to a polyvinylidene difluoride (PVDF) membrane (Millipore). The membrane was blocked by $5 \%$ nonfat milk for $1 \mathrm{~h}$ at room temperature, incubated overnight at $4{ }^{\circ} \mathrm{C}$ with primary antibodies against GPR18 (1:1000), nNOS (1:2000), eNOS (1:2000), ZO-1 (1:2000), PhosERK1/2 (1:1000), 5-LOX (1:2000), Phos-5-LOX (1:500), and $\beta$-Tubulin (1:5000), and then incubated with the indicated horseradish peroxidase (HRP)-conjugated secondary antibodies (Santa Cruz, 1:5000) for $2 \mathrm{~h}$ at room temperature. The band signal was detected using SuperSignal West Pico chemiluminescent substrate (Thermo Fisher Scientific). The protein quantification was analyzed by ImageJ software and normalized to that of the loading control. The phosphorylated level of proteins was normalized to its total protein level.

\section{Immunofluorescence staining}

The protein levels of nNOS and eNOS in neurons and BMVECs were detected by immunofluorescence staining. After incubation with the primary antibodies for nNOS, eNOS, NeuN, and vWF overnight at $4{ }^{\circ} \mathrm{C}$, the indicated secondary antibodies (Alexa Fluor 488 donkey anti-rabbit IgG antibody and Alexa Fluor 555 donkey anti-mouse IgG antibody) were incubated for $1 \mathrm{~h}$ at $37{ }^{\circ} \mathrm{C}$, followed by washing with PBST three times. Then, the sections were protected by cover slips with anti-fading mounting medium containing DAPI. Normal rabbit/mouse IgG served as the negative control for the immunofluorescence assay (data not shown). Finally, sections were observed by a fluorescence microscope (Olympus BX50/ BX-FLA/DP70), and the relative fluorescence intensity was analyzed by ImageJ software.

\section{TTC staining}

After $\mathrm{MCAO} / \mathrm{R}$ for $72 \mathrm{~h}$, the rats were sacrificed under anesthetization. The brains were removed and frozen quickly in precooling stainless-steel brain matrices $\left(-20^{\circ} \mathrm{C}\right)$ for $10 \mathrm{~min}$. Next, the brains were sliced coronally at $2 \mathrm{~mm}$ thickness for 5 slides starting from the frontal pole, and the cerebellum and olfactory bulb sections were discarded. Slices were then immersed in $2.0 \%$ TTC solution for $30 \mathrm{~min}$ at $37^{\circ} \mathrm{C}$, followed by washing three times with saline. Then the sections were fixed in $10 \%$ formalin and captured with a digital camera. Finally, we calculated the infarct volume as described previously [37]. In brief, the mean of the total infarction of each section was calculated as the average of the area on the rostral and the caudal side. Total infract volumes were calculated by adding the average area of each section and then multiplying by $2 \mathrm{~mm}$ (thickness of the sections). Infarct volume was also calculated as a percentage of the ipsilateral hemispheric volume by an investigator who was blinded to the group assignment.

\section{Drug administration}

The $\omega-3$ fatty acid pretreatment rats received an oral gavage of $30 \% \omega-3$ fatty acids at $1 \mathrm{~g} / \mathrm{kg}$ once every $24 \mathrm{~h}$ for the indicated time. RvD2 and O-1918 were dissolved in PBS and DMSO, respectively, at the stock concentration and diluted to $50 \mu \mathrm{g} / \mathrm{kg}$ and $3 \mu \mathrm{mol} / \mathrm{kg}$ by PBS per the manual, followed by intraperitoneal injection after the MCAO/R model was established for $72 \mathrm{~h}$. The RvD2 dosage was based on the research on RvD1 [38] and confirmed by experiment 1 (Fig. 1). In the in vitro experiment, RvD2 was used to treat cultured neurons, microglia, astrocytes, and BMVECs immediately after $\mathrm{OGD} / \mathrm{R}$ at $10 \mu \mathrm{mol} / \mathrm{L}$.

\section{Statistical analyses}

The frequency distribution for the neurobehavioral score assay was determined, and the neurobehavioral score was shown as the median, with an interquartile range. All other data were presented as mean \pm SEM and analyzed by one-way ANOVA followed by Dunnett's test. GraphPad Prism 7.00 was used for all statistical analysis. $P<0.05$ was considered to be a significant difference.

\section{Abbreviations \\ BBB: Blood-brain barrier; BMVEC: Brain microvascular endothelial cell; CNS: Central nervous system; CSF: Cerebrospinal fluid; EBI: Early brain injury; ELISA: Enzyme-linked immunosorbent assay; eNOS: Endothelial NOS; GPCR: G protein-coupled receptor; I/R: Ischemia/reperfusion; IL-6: Interleukin 6; LOX: Lipoxygense; MCAO: Middle cerebral artery occlusion; MCAO/R: Middle cerebral artery occlusion and reperfusion; nNOS: Neuronal NOS. \\ ROS: Reactive oxygen species; RvD2: Resolvin D2; SAH: Subarachnoid hemorrhage; TNF-a: Tumor necrosis factor a; ZO-1: Zonula occludens 1 \\ Acknowledgements \\ None. \\ Funding \\ This work was supported by supported by the Project of Jiangsu Provincial Medical Innovation Team (CXTDA2017003), Jiangsu Provincial Medical Youth Talent (QNRC2016728), Suzhou Key Medical Center (Szzx201501), grant from the National Natural Science Foundation of China, Scientific Department of Jiangsu Province (No. BL2014045), Suzhou Government (No. SYS201608, and LCZX201601), Jiangsu Province (No. 16KJB320008).}

Availability of data and materials Not applicable.

\section{Authors' contributions}

GZ and RM carried out the experiments. DZ participated in the design of the study and performed the statistical analysis. HS helped to draft the manuscript. XL participated in the acquisition of data. GC carried out analysis and interpretation of data. ZW participated in the design of the study. $\mathrm{HL}$ drafted the manuscript. All authors read and approved the final manuscript.

\section{Ethics approval}

All animal experiments are strictly in accordance with the guideline of Soochow University Institutional Animal Care and Use Committee. 


\section{Competing interests}

The authors declare that they have no competing interests.

\section{Publisher's Note}

Springer Nature remains neutral with regard to jurisdictional claims in published maps and institutional affiliations.

\section{Received: 7 September 2017 Accepted: 1 February 2018}

\section{Published online: 13 February 2018}

\section{References}

1. Bai Y, Y Zhang, B Han, L Yang, X Chen, R Huang, F Wu, J Chao, P Liu, G Hu, $\mathrm{JH}$ Zhang, and H Yao. Circular RNA DLGAP4 ameliorates ischemic stroke outcomes by targeting miR-143 to regulate endothelial-mesenchymal transition associated with blood-brain barrier integrity. J Neurosci. 2018; 38(1):32-50.

2. Cassella CR, Jagoda A. Ischemic stroke: advances in diagnosis and management. Emerg Med Clin North Am. 2017;35(4):911-30.

3. Reinhold AK, Rittner HL. Barrier function in the peripheral and central nervous system-a review. Pflugers Arch. 2017;469(1):123-34

4. Li H, Gao A, Feng D, Wang Y, Zhang L, Cui Y, Li B, Wang Z, Chen G. Evaluation of the protective potential of brain microvascular endothelial cell autophagy on blood-brain barrier integrity during experimental cerebral ischemia-reperfusion injury. Transl Stroke Res. 2014;5(5):618-26.

5. Jiang J, Luo Y, Qin W, Ma H, Li Q, Zhan J, Zhang Y. Electroacupuncture suppresses the NF-kappaB signaling pathway by upregulating cylindromatosis to alleviate inflammatory injury in cerebral ischemia/ reperfusion rats. Front Mol Neurosci. 2017;10:363.

6. Serhan CN, Hong S, Gronert K, Colgan SP, Devchand PR, Mirick G, Moussignac RL. Resolvins: a family of bioactive products of omega-3 fatty acid transformation circuits initiated by aspirin treatment that counter proinflammation signals. J Exp Med. 2002;196(8):1025-37.

7. Andreone BJ, Chow BW, Tata A, Lacoste B, Ben-Zvi A, Bullock K, Deik AA Ginty DD, Clish CB, Gu C. Blood-brain barrier permeability is regulated by lipid transport-dependent suppression of Caveolae-mediated transcytosis. Neuron. 2017;94(3):581-94. e5

8. Claria J, Dalli J, Yacoubian S, Gao F, Serhan CN. Resolvin D1 and resolvin D2 govern local inflammatory tone in obese fat. J Immunol. 2012;189(5):2597-605.

9. Wang X, Zhu M, Hjorth E, Cortes-Toro V, Eyjolfsdottir H, Graff C, Nennesmo I, Palmblad J, Eriksdotter M, Sambamurti K, Fitzgerald JM, Serhan CN, Granholm AC, Schultzberg M. Resolution of inflammation is altered in Alzheimer's disease. Alzheimers Dement. 2015;11(1):40-50. e1-2

10. Hashimoto M, Katakura M, Tanabe Y, Al Mamun A, Inoue T, Hossain S, Arita M, Shido $\mathrm{O}$. N-3 fatty acids effectively improve the reference memory-related learning ability associated with increased brain docosahexaenoic acid-derived docosanoids in aged rats. Biochim Biophys Acta. 2015;1851(2):203-9.

11. Chiang N, Fredman G, Backhed F, Oh SF, Vickery T, Schmidt BA, Serhan CN. Infection regulates pro-resolving mediators that lower antibiotic requirements. Nature. 2012:484(7395):524-8.

12. Norling LV, Dalli J, Flower RJ, Serhan CN, Perretti M. Resolvin D1 limits polymorphonuclear leukocyte recruitment to inflammatory loci: receptordependent actions. Arterioscler Thromb Vasc Biol. 2012;32(8):1970-8.

13. Mishina M, Kim K, Kominami S, Mizunari T, Kobayashi S, Katayama Y. Impact of polyunsaturated fatty acid consumption prior to ischemic stroke. Acta Neurol Scand. 2013;127(3):181-5.

14. Yin J, Li H, Meng C, Chen D, Chen Z, Wang Y, Wang Z, Chen G. Inhibitory effects of omega-3 fatty acids on early brain injury after subarachnoid hemorrhage in rats: possible involvement of $\mathrm{G}$ protein-coupled receptor 120/beta-arrestin2/TGF-beta activated kinase-1 binding protein-1 signaling pathway. Int J Biochem Cell Biol. 2016;75:11-22.

15. Gilbert NC, Rui Z, Neau DB, Waight MT, Bartlett SG, Boeglin WE, Brash AR, Newcomer ME. Conversion of human 5-lipoxygenase to a 15-lipoxygenase by a point mutation to mimic phosphorylation at Serine-663. FASEB J. 2012; 26(8):3222-9.

16. Finlay DB, Joseph WR, Grimsey NL, Glass M. GPR18 undergoes a high degree of constitutive trafficking but is unresponsive to N-Arachidonoyl Glycine. PeerJ. 2016;4:e1835.

17. Hong S, Lu Y, Yang R, Gotlinger KH, Petasis NA, Serhan CN. Resolvin D1, protectin D1, and related docosahexaenoic acid-derived products: analysis via electrospray/low energy tandem mass spectrometry based on spectra and fragmentation mechanisms. J Am Soc Mass Spectrom. 2007;18(1):128-44.
18. Deyama S, Y Ishikawa, K Yoshikawa, K Shimoda, S Ide, M Satoh, and M Minami. Resolvin D1 and D2 reverse lipopolysaccharide-induced depressionlike behaviors through the MTORC1 signaling pathway. Int J Neuropsychopharmacol. 2017;20(7):575-84.

19. Park CK, Xu ZZ, Liu T, Lu N, Serhan CN, Ji RR. Resolvin D2 is a potent endogenous inhibitor for transient receptor potential subtype V1/A1, inflammatory pain, and spinal cord synaptic plasticity in mice: distinct roles of resolvin D1, D2, and E1. J Neurosci. 2011;31(50):18433-8.

20. Chiang N, Dalli J, Colas RA, Serhan CN. Identification of resolvin D2 receptor mediating resolution of infections and organ protection. J Exp Med. 2015; 212(8):1203-17.

21. Gantz I, Muraoka A, Yang YK, Samuelson LC, Zimmerman EM, Cook H, Yamada T. Cloning and chromosomal localization of a gene (GPR18) encoding a novel seven transmembrane receptor highly expressed in spleen and testis. Genomics. 1997:42(3):462-6.

22. McHugh D. GPR18 in microglia: implications for the CNS and endocannabinoid system signalling. Br J Pharmacol. 2012;167(8):1575-82.

23. Xu ZZ, Zhang L, Liu T, Park JY, Berta T, Yang R, Serhan CN, Ji RR. Resolvins RvE1 and RvD1 attenuate inflammatory pain via central and peripheral actions. Nat Med. 2010;16(5):592-7. 1 p following 597

24. Forstermann U, Sessa WC. Nitric oxide synthases: regulation and function. Eur Heart J. 2012:33(7):829-37. 837a-837d

25. Alexander SP. So what do we call GPR18 now? Br J Pharmacol. 2012;165(8): 2411-3.

26. Liu W, Wang P, Shang C, Chen L, Cai H, Ma J, Yao Y, Shang X, Xue Y. Endophilin-1 regulates blood-brain barrier permeability by controlling ZO-1 and occludin expression via the EGFR-ERK1/2 pathway. Brain Res. 2014;1573:17-26.

27. Bacarin CC, de Sa-Nakanishi AB, Bracht A, Matsushita M, Santos Previdelli I, Mori MA, de Oliveira RM, Milani H. Fish oil prevents oxidative stress and exerts sustained antiamnesic effect after global cerebral ischemia. CNS Neurol Disord Drug Targets. 2015;14(3):400-10

28. Bas O, Songur A, Sahin O, Mollaoglu H, Ozen OA, Yaman M, Eser O, Fidan H, Yagmurca M. The protective effect of fish n-3 fatty acids on cerebral ischemia in rat hippocampus. Neurochem Int. 2007;50(3):548-54.

29. Kavakli A, Kose E, Kus I, Zararsiz I, Akpolat N, Sarsilmaz M. Protective effect of omega-3 fatty acids in a rat focal cerebral ischemia-reperfusion model. Neurosciences (Rivadh). 2007:12(3):198-201.

30. Correia Bacarin C, Mori MA, Dias Fiuza Ferreira E, Valerio Romanini C, Weffort de Oliveira RM, Milani H. Fish oil provides robust and sustained memory recovery after cerebral ischemia: influence of treatment regimen. Physiol Behav. 2013;119:61-71.

31. Yang DY, Pan HC, Yen YJ, Wang CC, Chuang YH, Chen SY, Lin SY, Liao SL, Raung SL, Wu CW, Chou MC, Chiang AN, Chen CJ. Detrimental effects of post-treatment with fatty acids on brain injury in ischemic rats. Neurotoxicology. 2007;28(6):1220-9.

32. Pascoal LB, Bombassaro B, Ramalho AF, Coope A, Moura RF, Correa-da-Silva F, Ignacio-Souza L, Razolli D, de Oliveira D, Catharino R, Velloso LA. Resolvin RvD2 reduces hypothalamic inflammation and rescues mice from dietinduced obesity. J Neuroinflammation. 2017;14(1):5.

33. Obenaus A, Ashwal S. Neuroimaging of stroke and ischemia in animal models. Transl Stroke Res. 2012;3(1):4-7.

34. Wang Y, Gao A, Xu X, Dang B, You W, Li H, Yu Z, Chen G. The Neuroprotection of Lysosomotropic agents in experimental subarachnoid hemorrhage probably involving the apoptosis pathway triggering by Cathepsins via chelating Intralysosomal iron. Mol Neurobiol. 2015;52(1):64-77.

35. Irvine HJ, AC Ostwaldt, MB Bevers, S Dixon, TW Battey, BC Campbell, SM Davis, GA Donnan, KN Sheth, R Jahan, JL Saver, CS Kidwell, and WT Kimberly. Reperfusion after ischemic stroke is associated with reduced brain edema. J Cereb Blood Flow Metab. 2017:271678X17720559.

36. Shen H, Liu C, Zhang D, Yao X, Zhang K, Li H, Chen G. Role for RIP1 in mediating necroptosis in experimental intracerebral hemorrhage model both in vivo and in vitro. Cell Death Dis. 2017;8(3):e2641.

37. Andrabi SA, Sayeed I, Siemen D, Wolf G, Horn TF. Direct inhibition of the mitochondrial permeability transition pore: a possible mechanism responsible for anti-apoptotic effects of melatonin. FASEB J. 2004;18(7):869-71.

38. Wang CS, Maruyama CL, Easley JT, Trump BG, Baker OJ. AT-RvD1 promotes resolution of inflammation in NOD/ShiLtJ mice. Sci Rep. 2017;7:45525. 